\title{
G-Quadruplexes in the Biology of Eukaryotic Cells
}

\section{Nora B. Calcaterra*}

Institute of Molecular and Cell Biology of Rosario (IBR), National Research Council (CONICET)-Faculty of Biochemical and Pharmaceutical Sciences, Universidad Nacional de Rosario,, Santa Fe-Argentina

According to the model proposed by James D. Watson and Francis Crick in 1953, the DNA molecule consists of two polynucleotide chains wound around each other to form a clockwise double helix. This structure is known as B form of DNA, B-DNA, and is the predominant conformation adopted by the DNA under relaxed conditions. However, the DNA molecule can transiently adopt other non-B or non-canonical structures. How these non-B DNA structures are folded in vivo and, what are the cellular conditions that favor the folding of such alternative structures are key questions for fully understanding the role of DNA structural polymorphism in biological processes.

In the past few decades a large number of scientific papers, both computational and experimental in vitro and in vivo, have demonstrated that G-rich DNA sequences can fold as intrastrand quadruple helix structures called G-quadruplexes or G4-DNA. G4-DNA is stabilized by G-quartets, planar arrays of four guanines paired by Hoogsteen hydrogen bonding, and monovalent alkali cation, $\mathrm{K}^{+}$or $\mathrm{Na}^{+}$, located in the central cavity of the structure.

In the human genome the sites that can potentially form G4DNA are estimated to be more than 3,000,000. They are not randomly distributed, but located preferentially in repetitive genomic sequences such as the telomeres, ribosomal DNA and the immunoglobulin heavy-chain switch regions. Moreover, G-rich sequences have been found with a high frequency in the control regions of proto-oncogenes, either upstream or downstream the transcription start site. Thus, G-quadruplexes have become potential targets for anticancer drugs, and several groups have attempted to identify small organic molecules that bind to them. Despite a few of the new anticancer drugs which have entered preclinical or clinical trials, the selectivity of these compounds has yet to be improved. As drug design targets, the topology and structural characteristics of G-quadruplexes, their possible biological roles, and the modes and sites of small-ligand binding to these structures should be understood clearly.

A prerequisite for the formation of G-quadruplexes is the destabilization of the B-DNA double-strand helix, which is highly stable. It is known that transcription, replication and other biological processes generate high levels of supercoiling, which can be propagated along the DNA-helix until susceptible sites wherein structural transitions may take place. Thus, the opening of the double stranded DNA either by transcription or replication bubble movement along the helix or due to the accumulation and propagation of torsional stress may transiently exposed single strand segments able to fold as G-quadruplexes structures.

Unlike tandem repeats present in the human telomeric sequences, G-rich sequences in gene promoters may contain more than four stretches of $G$ and, thus, each of these segments is unique in the number of $\mathrm{G}$ residues that form and in the length of the connectors that originate nucleotide residue loops. This results in a mixture of conformational isomers in a multiple dynamic equilibrium. In a particular cellular context, specific proteins could associate with a subpopulation of such polymorphic structures and, thus, shift the equilibrium toward particular conformational isomers. Thus, the G-quadruplex polymorphism could function as a molecular switch enabling gene expression modulation by transitions in DNA structure.

Of note, several proteins were identified associated with the putative G-quadruplex motifs; most of them are highly conserved zincfinger binding proteins. Because the versatility of zinc-finger binding pocket is remarkable, it is interesting to consider the implications of G-quadruplex-zinc-finger interactions as a pair. In this context, variations in both DNA polymorphism and protein domains may contribute to a fine tuning of transcriptional control.

Ontology studies revealed that genes containing promoters with high potential to fold as G-quadruplexes are related to defined processes, such as development, neurogenesis and cell proliferation/ cell-cycle regulation. However, scant information has been gathered so far about the role of G-quadruplex and its associated proteins in these processes. This issue has become one of the major challenges for scientists interested in fully understanding the role of G-quadruplexes in eukaryotic biology. Pharmaceutical Sciences, National University of Rosario, Suipacha 531, (S2002LRK) Rosario, Santa Fe-Argentina, E-mail: calcaterra@ibr.gov.ar

Received August 23, 2012; Accepted August 24, 2012; Published August 26 2012

Citation: Calcaterra NB (2012) G-Quadruplexes in the Biology of Eukaryotic Cells Mol Biol 1:e103. doi:10.4172/2168-9547.1000e103

Copyright: ( 2012 Calcaterra NB. This is an open-access article distributed unde the terms of the Creative Commons Attribution License, which permits unrestricted use, distribution, and reproduction in any medium, provided the original author and source are credited. 